\title{
LÉXICO SANMARQUINO: ESTUDIO LEXICOGRÁFICO PRELIMINAR
}

\author{
Sanmarquino lexicon: preliminary lexicographic study
}

\section{Luisa Portilla-Durand ${ }^{1}$}

\author{
A los estudiantes de pregrado de San Marcos, \\ a la espera de que termine la pandemia \\ y puedan volver nuevamente a las aulas.
}

Resumen

Haensch y otros señalan que la lexicografía «está estrechamente relacionada con la vida humana en sus aspectos más variados», por lo que es la disciplina «que mayores servicios presta a la colectividad» (1982, p. 12). Así, con en el estudio preliminar del LÉXICO SANMARQUINO se busca evidenciar el aporte de la Lexicografía no solo a la investigación lingüística sino también a la investigación histórica y social.

Los diccionarios ofrecen «acervos léxicos, que sin lugar a dudas son el elemento central de la riqueza de las lenguas y de la posibilidad humana de conservar la memoria de sus experiencias compartidas» (Lara, 2006, p. 144). En tal sentido, si los repertorios léxicos de cualquier índole no se registran, se pierde la posibilidad de conocer la riqueza de las lenguas y se pierde también la memoria de las experiencias compartidas por sus hablantes.

En el LÉXICO SANMARQUINO, a partir de conceptos de la Lexicografía moderna, se aborda el estudio lexicográfico de la lengua juvenil, se proponen definiciones y se identifican algunos procesos de formación del léxico coloquial usado por los estudiantes sanmarquinos de pregrado.

Palabras clave: léxico sanmarquino, Lexicografía, lengua juvenil, léxico coloquial

1 Lingüista. Profesora Principal de la Universidad Nacional Mayor de San Marcos. Miembro A del Instituto de Investigaciones del Pensamiento Peruano y Latinoamericano (IIPPLA) de la UNMSM, Lima, Perú.

\begin{abstract}
Haensch and others point out that Lexicography is closely related to human life in its most varied aspects, making it the discipline that provides the greatest services to the community (1982). Thus, with the preliminary study of the SANMARQUINO LEXICON, the aim is to demonstrate the contribution of Lexicography not only to linguistic research but also to historical and social research.

Dictionaries offer lexical collections, which are undoubtedly the central element of the richness of languages and the human possibility of preserving the memory of their shared experiences (Lara, 2006). In this regard, if lexical repertoires of any kind are not recorded, the possibility of knowing the richness of languages is lost and the memory of the experiences shared by their speakers is also lost. In the SANMARQUINO LEXICON, based on the concepts of modern Lexicography, the lexicographic study of the juvenile language is carried out, definitions are proposed and some processes of formation of the colloquial lexicon used by San Marcos undergraduate students are identified.
\end{abstract}

Keywords: Sanmarquino lexicon, Lexicography, juvenile language, colloquial lexicon

\section{INTRODUCCIÓN}

D e acuerdo con Haensch y otros, la Lexicografía es «quizás, entre todas las actividades lingüísticas, junto con la traducción y la enseñanza de idiomas, la que 
está estrechamente relacionada con la vida humana en sus aspectos más variados y la que mayores servicios presta a la colectividad» (1982, p. 12).

Por su parte, Lara señala que el diccionario «materializa el léxico y por eso se convierte en un vehículo privilegiado de la reflexión y de la interpretación de la manera en que se articula la lengua con la sociedad, con su historia y con su tradición» (1990, pp. 136-137).

Siguiendo la línea conceptual de Luis Hernán Ramírez (1996), el LÉXICO SANMARQUINO se definiría como un tipo de lengua juvenil, es decir, una lengua especial cerrada que utilizan los estudiantes sanmarquinos de pregrado y con la cual se «pretende ocultar la comunicación de los más jóvenes a las personas de otras generaciones» (ibídem, p. 150). La pregunta sería, entonces, cómo quienes no forman parte del grupo de estudiantes sanmarquinos de pregrado pueden acceder al conocimiento de esta lengua especial. Luis Hernán Ramírez permite responder a la pregunta: puesto que la lengua juvenil no tiene carácter delictivo, el entorno puede acceder al conocimiento de las voces de la lengua especial, aunque, como la intención de los usuarios de esta lengua es «mantenerla en secreto», el vocabulario de la lengua juvenil es muy cambiante, por lo que «su principal característica es la gran movilidad de su léxico»(loc. cit.). De allí que pueda anticiparse que las voces del LÉxICO SANMARQUINO cambiarán con el tiempo en cuanto a forma y/o acepción.

MARCO CONCEPTUAL Y SU APLICACIÓN EN EL LÉXICO SANMARQUINO

Respecto de la estructuración del LÉXICO SANMARQUINO, se deben señalar dos componentes fundamentales: la macroestructura y la microestructura. Martínez de Sousa
(1995, p. 259) define la macroestructura de la siguiente manera: «Conjunto de las entradas seleccionadas para formar un diccionario»; mientras que la microestructura la define así: "Conjunto de informaciones ordenadas que en el artículo lexicográfico siguen a la entrada» (ibídem, p. 275). En cuanto a la entrada lexicográfica, esta «encabeza un artículo de diccionario, vocabulario, glosario, terminología» (ibídem, p. 180); mientras que las subentradas son aquellas «entradas sintagmáticas que forman parte del artículo del que es cabeza la entrada» (ibídem, p. 315). El LÉXICO SANMARQUINO está compuesto por entradas y subentradas, y ambas se presentan en negrita en el artículo lexicográfico. El artículo lexicográfico puede ser de dos clases: artículo léxico, en el que se define una palabra léxica: sustantivos, adjetivos, verbos, o artículo gramatical, en el que se explica una palabra gramatical: artículos, conjunciones, preposiciones, adverbios (ibídem, p. 41), y también puede ser clasificado como simple si presenta una sola definición, o complejo si presenta más de una (loc. cit.). El LÉXICO SANMARQUINO está compuesto por artículos léxicos tanto simples como complejos. Por otra parte, la definición lexicográfica es «todo tipo de equivalencia establecida entre la entrada y cualquier expresión explicativa de la misma en un diccionario monolingüe» (Porto Dapena, 2002, p. 269), siempre que se establezca «una relación entre tres términos: el definido, el genérico (o descriptor) y el diferenciador o especificador» (Martínez de Sousa, 1995, p. 76). Esta acotación es relevante a fin de distinguir la definición lexicográfica de la definición sinonímica, en la que se define por remisión a otra palabra que comparte la misma acepción, y de la definición enciclopédica que se usa en especial para definir animales y plantas, ya que en estos casos se requiere enumerar "partes, tamaños, formas, etc., en cantidad necesaria para distinguir lo definido de cualquier otro 
término que se le pueda parecer» (ibídem, p. 74). En el LÉXICO SANMARQUINO se presentan tanto definiciones sinonímicas, lexicográficas como enciclopédicas. Respecto de las definiciones, en particular las lexicográficas, debe considerarse el contenido: "Conjunto de elementos que ofrecen información fundamental en una definición», y el contorno: «Conjunto de elementos de información no esencial añadidos a una definición» (ibídem, p. 68). En el LÉXICO SANMARQUINO, el contorno se presenta antes de la definición seguido de dos puntos. Ya sea en las definiciones lexicográficas, sinonímicas o enciclopédicas es posible hacer uso de la remisión, es decir, valerse de la definición de una palabra para proponer la definición de otra; de acuerdo con ello existen dos tipos de remisión: endofórica, cuando se utiliza la definición de una palabra que se halla en el propio diccionario o léxico del cual se trata (remisión interna); exofórica, cuando se utiliza la definición de una palabra que se halla en un diccionario o léxico distinto del que se trata (remisión externa). En el LÉXICO SANMARQUINO se hace uso de la remisión exofórica, en específico respecto del Diccionario de la lengua española (DLE) 2014.

En cuanto al nivel de uso, todas las voces del LÉXICO SANMARQUINO corresponden al nivel de uso coloquial: modalidad «utilizada por los hablantes en sus relaciones cotidianas; se la emplea en todos los menesteres diarios de la comunicación, en el círculo de nuestras amistades más íntimas, con las personas en quienes depositamos una gran confianza y ante quienes no nos sentimos tan obligados a la observancia rígida e irrestricta de las normas de cortesía» (Ramírez, 1996, p. 133). Por corresponder las voces del LÉxICO SANMARQUINO al nivel de uso coloquial, se caracterizan por presentar «abreviaciones y otros cambios así como por el manejo de un léxico común semánticamente polivalente y rico en sentidos figurados y traslaticios» (ibídem, pp. 133-134), por lo que muchos términos «no son más que traslaciones semánticas de las palabras de uso general» (ibídem, p. 134). Lo señalado permite explicar las analogías fonéticas, los acortamientos, la composición, entre otros, así como las metonimias y metáforas presentes en el LÉXICO SANMARQUINO, procesos similares a los observados en la formación del léxico popular limeño (Portilla, 2011). Por tratarse de un estudio lexicográfico preliminar, solo se explicitan algunos casos de formación de palabras del LÉXICO SANMARQUINO y, por ello también, los procesos semánticos (metonimia y metáfora) se analizarán en otro estudio.

\section{ENTRADAS, SUBENTRADAS Y DEFINICIONES}

\section{DEL LÉXICO SANMARQUINO}

En el LÉXICO SANMARQUINO, las entradas siguen el orden alfabético internacional, es decir, las letras $c h$ y $l l$ se incluyen en la $c$ y en la $l$, respectivamente. La información etimológica se coloca inmediatamente después de la entrada y solo en los casos en los que se requiere explicitar el símbolo de copyright (C). Las marcas gramaticales se colocan siempre después de cada entrada o subentrada, y estas se enumeran solo cuando existe más de una. En el LÉXICO SANMARQUINO no se marca el nivel de uso, pues todas las voces corresponden al nivel de uso coloquial. La doble pleca (\|) se utiliza para separar acepciones o para separar la entrada de una subentrada; también se usa en las remisiones exofóricas, cuando se presenta el resumen de una acepción que se encuentra en el DLE 2014. Las formas sinonímicas, si las hubiera, se presentan en cursivas al final de cada definición antecedidas por una flecha con cabeza hacia la izquierda $(\longleftarrow)$. Asimismo, al final de algunas definiciones se presentan Notas explicativas respecto de la entrada o subentrada, o respecto de la acepción. (Las NotAs no se presentan con regularidad debido 
a que se trata de un estudio preliminar.)

\section{A}

académico, ca. m. y f. Alumno muy estudioso.

ADM. com. Estudiante de la Escuela Profesional de Administración. NotA. La entrada ADM se forma por siglación con los componentes iniciales de la palabra adm(inistración).

alumno, na. $\|$ alumno eterno. $\mathrm{m}$. Alumno que cursa estudios de pregrado por más de cinco años. $\leftarrow$ cachimbo viejo, dinosaurio, fáder, patriarca, supercachimbo

antropófago, ga. $\mathrm{m}$. y f. Estudiante de la Escuela Profesional de Antropología. NotA. La palabra antropófago presenta semejanza fonética con Antropología.

anunciación. || lugar de la anunciación. m. Parque ubicado a la espalda del Rectorado, donde concurren las parejas de enamorados por las tardes o por las noches. $\leftarrow$ grito de las virgenes

apoyo. m. Generalmente en un papel pequeño: Texto que sirve como plagio.

atajo. \| atajo de crash. (@) Del videojuego Crash Bandicoot.) m. Para acortar un tramo: Camino que se encuentra entre la Escuela Profesional de Mecánica de Fluidos y la Escuela Profesional de Ingeniería Electrónica. Nota. Crash Bandicoot es el nombre de una serie de videojuegos protagonizada por un personaje del mismo nombre.

autista. com. Estudiante muy callado y solitario.

B

base. f. Año de ingreso a la universidad.

buitre. com. Estudiante que vive en la residencia universitaria. $\leftarrow$ parásito

búnquer. m. Facultad de Ingeniería Mecánica de Fluidos. burgués, sa. 1. m. y f. Estudiante de la Facultad de Economía. \| 2. m. y f. Estudiante a quien no le agrada ir a comer al comedor universitario.

burguesía. || secuaz de la burguesía. com. Estudiante de la Facultad de Derecho y Ciencia Política.

burrear. intr. Viajar en los buses de la UNMSM.

burro. m. Bus de la UNMSM. \| señor burro. m. Chofer del bus de la UNMSM.

C

cachimbear. tr. Tratar mal a un ingresante. cachimbero. $\mathrm{m}$. Estudiante que enamora a las cachimbas.

cachimbo, ba. m. y f. Alumno que acaba de ingresar a la universidad y que, por tanto, cursa las asignaturas del primer semestre de pregrado. || cachimbo viejo. $\mathrm{m}$. Alumno que cursa estudios de pregrado por más de cinco años. $\leftarrow$ alumno eterno, dinosaurio, fáder, patriarca, supercachimbo

cachimbombo. m. Cachimbo de conducta afeminada. NotA. La palabra cachimbombo se forma a partir de la composición de cachimbo $>$ cachim >ca + chimbombo ('homosexual'). Se usa en sentido despectivo.

cálculo. || cálculo renal. m. Curso difícil relacionado con cálculos matemáticos.

cangallear. intr. Ir a comer al comedor universitario de Cangallo.

cangalloman. $\mathrm{m}$. Estudiante que siempre va a comer al comedor de Cangallo y que es resistente a las afecciones estomacales. NотA. La palabra cangalloman se forma a partir de la composición de cangallo + man ('hombre', en inglés).

carroña. f. Comida que se sirve en el comedor universitario. $\leftarrow$ muerte lenta

carroñear. intr. Comer en el comedor universitario. $\leftarrow$ gusanear 
carroñero, ra. m. Estudiante que come en el comedor universitario. $\leftarrow$ gusano, parásito

cavernícola. m. Estudiante que desconoce las innovaciones tecnológicas. $\leftarrow$ oxidado

cementerio. || cementerio de los burros. m. Lugar descampado, dentro de la UNMSM, donde se dejan los buses sanmarquinos que están malogrados.

chismoso, sa. m. y f. Estudiante de la Escuela Profesional de Historia. $\leftarrow$ chistórico, cuentista

chistórico, ca. m. y f. Estudiante de la Escuela Profesional de Historia. $\leftarrow$ chismoso, cuentista

compañebrio. m. Compañero de estudios que bebe licor con frecuencia. NotA. La palabra compañebrio se forma a partir de la composición de compañero > compañ + ebrio.

contemplativo, va. m. y f. Alumno que tiene muchos conocimientos, pero que no los pone en práctica. $\leftarrow$ teórico de gabinete

cuentista. com. Estudiante de la Escuela Profesional de Historia. $\leftarrow$ chismoso, chistórico

D

dinosaurio. m. Alumno que cursa estudios de pregrado por más de cinco años. $\leftarrow$ alumno eterno, cachimbo viejo, fáder, patriarca, supercachimbo

dobletear. intr. Recibir doble ración en el comedor universitario.

doctorado, da. m. y f. Estudiante que desaprueba varias veces un curso. $\leftarrow$ magíster

E

eléctrico, ca. m. y f. Estudiante de la Facultad de Ingeniería Electrónica y Eléctrica.

examen. \| examen basura. m. Examen muy difícil.
$\mathbf{F}$

fáder. m. Alumno que cursa estudios de pregrado por más de cinco años. $\leftarrow$ alumno eterno, cachimbo viejo, dinosaurio, patriarca, supercachimbo Nota. La palabra fáder es un calco del inglés father ('padre').

fantástico, ca. m. Profesor que enseña un curso para el que no está preparado.

fiqui. m. Curso de Físico-Química. NotA. La palabra fiqui se forma por composición a partir de los componentes iniciales de físico y química: $f i+q u i \rightarrow$ fiqui.

forralibros. com. Estudiante de la Escuela Profesional de Bibliotecología.

G

gabinete. || teórico de gabinete. $1 . \mathrm{m}$. Estudiante que tiene muchos conocimientos, pero que no los pone en práctica. $\leftarrow$ contemplativo $\|$ 2. m. Estudiante que pasa mucho tiempo estudiando en la biblioteca. $\leftarrow$ sinamigos

grito. || grito de las vírgenes. $\mathrm{m}$. Parque ubicado a la espalda del Rectorado, donde concurren las parejas de enamorados por las tardes o por las noches. $\leftarrow$ lugar de la anunciación

gusanear. intr. 1. Hacer una cola, con las mochilas como señal, para comer en el comedor universitario. || 2. intr. Comer en el comedor universitario. $\leftarrow$ carroñear

gusano. 1. m. Estudiante que come en el comedor universitario. $\leftarrow$ carroñero, parásito $\|$ 2. m. Cola que se hace, con las mochilas como señal, para comer en el comedor universitario.

H

habitación. || ir a la habitación del tiempo. (C) Del manga Dragon Ball.) loc. verb. Antes de un examen o de una exposición: Aislarse en un ambiente de la Universidad para estudiar 
a última hora. NotA. En el manga Dragon Ball, la Habitación del Tiempo es un lugar en el que un día de entrenamiento equivale a un año y donde se puede mejorar con creces la fuerza, velocidad, resistencia, etc.

huaquero, ra. m. y f. Estudiante de la Escuela Profesional de Arqueología.

hueco. m. Tiempo libre entre un curso y otro.

huevología. m. Curso fácil. Nota. La palabra huevología se forma a partir de la composición de huevo + -logía ('estudio'). Respecto de huevo, en el DA 2010 se encuentra la siguientre acepción para el uso peruano: «Referido a una tarea, fácil de cumplir» (p. 1165).

\section{I}

inicialeca. com. Estudiante de la especialidad de Educación Inicial. NotA. La entrada inicialeca es un compuesto formado a partir de las palabras inicial + leca ('lesbiana'). Se usa en sentido despectivo.

interrogatorio. m. Examen sustitutorio. $\leftarrow$ lloratorio

intoxicado, da. m. y f. Estudiante de la Escuela Profesional de Ingeniería de Minas. $\leftarrow$ topo

K

ki. [...] || elevar el ki. (C) Del manga Dragon Ball.) loc. verb. Entrenarse resolviendo ejercicios de cálculo matemático con la finalidad de adquirir destreza para solucionarlos. NotA. En el manga Dragon Ball, el ki es la energía vital.

L

lamento. || muro de los lamentos. m. Lugar donde se publican los resultados del examen de admisión.

lloratorio. m. Examen sustitutorio. $\leftarrow$ interrogatorio lobo, ba. m. y f. Estudiante que tiene mucho conocimiento de uno o más cursos. Nota. Se usa también en sentido despectivo.

loco, ca. m. y f. Estudiante de la Escuela Profesional de Psicología.

lotear. intr. Jugar un partido de fútbol. NotA. La entrada lotear se deriva del acortamiento de pelotear (aféresis: acortamiento del inicio de palabra).

M

mafioso, sa. m. y f. Estudiante que acostumbra a plagiar.

magíster. com. Estudiante que desaprueba varias veces un curso. $\leftarrow$ doctorado

mandraquear. tr. Acomodar los datos para que cuadre un informe técnico.

matadero. m. Comedor universitario. $\leftarrow$ muerte lenta

matagente. m. Profesor que desaprueba a muchos alumnos.

menú. || menú frío. m. Menú que consta de galleta, atún y gaseosa o jugo de frutas.

merca. m. Examen de ciclos anteriores que se usa como plagio.

metalero, ra. m. y f. Estudiante de la Escuela Profesional de Ingeniería Metalúrgica.

microbio. com. Estudiante de la Escuela Profesional de Microbiología y Parasitología.

muerte. || muerte lenta. 1. f. Comedor universitario. $\leftarrow$ matadero $\| 2$. f. Comida que se sirve en el comedor universitario. $\leftarrow$ carroña muerte viviente. f. Estudiante de la Facultad de Ciencias Matemáticas. $\leftarrow$ sinvida

$\mathbf{N}$

nerd. com. Alumno a quien se considera de alto coeficiente intelectual, dedicado al estudio y poco sociable. NotA. Se usa también en sentido despectivo. 
$\mathbf{O}$

ociólogo, ga. m. y f. Estudiante de la Escuela Profesional de Sociología. NotA. La entrada ociólogo se deriva del acortamiento de sociólogo (aféresis: acortamiento del inicio de palabra); se tiene, entonces, ocio $+-\log o$ : si el sociólogo es el especialista en Sociología, el ociólogo sería el «especialista en el ocio».

oxidado, da. m. y f. Estudiante que desconoce las innovaciones tecnológicas. $\leftarrow$ cavernícola

$\mathbf{P}$

panadero, ra. m. y f. Estudiante de la Facultad de Ingeniería Industrial.

papá. [...] || Papá Noel. m. Profesor que aprueba a todos los alumnos.

parásito. 1. m. Estudiante que vive en la residencia universitaria. $\leftarrow$ buitre $\|$ 2. m. Estudiante que come en el comedor universitario. $\leftarrow$ carroñero, gusano

patriarca. m. Alumno que cursa estudios de pregrado por más de cinco años. $\leftarrow$ alumno eterno, cachimbo viejo, dinosaurio, fater, supercachimbo

pesadilla. m. examen (\| prueba para demostrar el aprovechamiento en los estudios) (DLE, 2014, p. 986).

plan. [...] || plan h. Pérdida de tiempo. NotA. En la subentrada plan $h, h$ se refiere a hueveo, que en el DA 2010 se define de la siguiente manera para el uso peruano: «Pérdida de tiempo al rehuir el trabajo» (p. 1164).

plancha. f. plagio ( $\|$ acción de plagiar) (DLE, 2014, p. 1728).

planchar. tr. plagiar (\|copiar en lo sustancial obras ajenas). (DLE, 2014, p. 1728).

planchero, ra. 1. m. y f. Estudiante que plagia en los exámenes. $\leftarrow$ práctico $\| 2$. m. y f. Profesor que transcribe literalmente parte de un libro en la pizarra. $\leftarrow$ profesor plancha práctico, ca. $\mathrm{m}$. y f. Estudiante que plagia en los exámenes. $\leftarrow$ planchero

primarioso, sa. m. y f. Estudiante de la especialidad de Educación Primaria.

pro. [...] || el, la más pro. $m$. y f. En un grupo: Estudiante que tiene más conocimientos.

profesor, ra. [...] || profesor picapiedra. $\mathrm{m}$. Profesor que desconoce las innovaciones tecnológicas. || profesor plancha. m. Profesor que transcribe literalmente parte de un libro en la pizarra. $\leftarrow$ planchero

$\mathbf{R}$

radical. com. Estudiante de la Facultad de Ciencias Sociales. $\leftarrow$ rogelio, rojo, tirapiedras

raro, ra. 1. m. y f. Estudiante de la Escuela Profesional de Filosofía. ||2. m. y f. Estudiante de la Escuela Profesional de Arte.

ratonear. intr. Ir a la biblioteca.

revisión. f. Acción de revisar los exámenes tomados a bases anteriores.

rogatorio. m. Examen sustitutorio. $\leftarrow$ suplicatorio

rogelio, lia. m. y f. Estudiante de la Facultad de Ciencias Sociales. $\leftarrow$ radical, rojo, tirapiedras. NotA. La palabra rogelio presenta semejanza fonética con rojo ('izquierdista').

rojo, ja. m. y f. Estudiante de la Facultad de Ciencias Sociales. $\leftarrow$ radical, rogelio, tirapiedras

S

sinamigos. com. Estudiante que pasa mucho tiempo estudiando en la biblioteca. $\leftarrow$ teórico de gabinete

sinvida. com. Estudiante de la Facultad de Ciencias Matemáticas. $\leftarrow$ muerte viviente

supercachimbo, ba. m. y f. Alumno que cursa estudios de pregrado por más de cinco años. $\leftarrow$ alumno eterno, cachimbo viejo, dinosaurio, fáder, patriarca

suplicatorio. m. Examen sustitutorio. $\leftarrow$ rogatorio 
$\mathbf{T}$

tigre. m. Respecto del desempeño en un curso: Estudiante que destaca.

tirapiedras. com. Estudiante de la Facultad de Ciencias Sociales. $\leftarrow$ radical, rogelio, rojo

topo.m. Estudiante delaEscuela Profesional de Ingeniería de Minas. $\leftarrow$ intoxicado

tripletar. intr. Recibir triple ración en el comedor universitario.

trol. com. Estudiante que es muy fastidioso. NotA. El término trol se usa en internet para aludir a una persona de identidad desconocida que publica mensajes para molestar a los lectores o para generar discordia entre ellos.

turista. com. Estudiante que falta a clases o que asiste irregularmente.

V

vitamina. f. Mezcla de diferentes bebidas alcohólicas.

$\mathbf{Y}$

Yepeto. m. Profesor anciano. NotA. En Las aventuras de Pinocho, novela de Carlo Collodi, Gepeto es un carpintero anciano que, como no tiene hijos, hace un muñeco de madera al que llama Pinocho.

\section{A Modo de CONClusión}

Los diccionarios han «ampliado de una manera vertiginosa la memoria colectiva del léxico, hasta el punto de superar las memorias individuales y ofrecernos grandes acervos léxicos, que sin lugar a dudas son el elemento central de la riqueza de las lenguas y de la posibilidad humana de conservar la memoria de sus experiencias compartidas» (Lara, 2006, p. 144).

Al estudiar el LÉXICO SANMARQUINO se sigue esta línea de pensamiento: superar las memorias individuales a fin de ampliar la memoria colectiva respecto del léxico peruano, en específico respecto de «un nivel de lengua fundamentalmente innovador y neológico» (Ramírez, 1996, p. 134), como lo es la lengua juvenil utilizada por los estudiantes sanmarquinos de pregrado, que presenta procesos fonéticos, morfológicos y semánticos innovadores, algunos de los cuales se han mostrado aquí, a excepción de los procesos semánticos.

Asimismo, en el estudio se pone en evidencia, una vez más, la riqueza del español y se aporta a la conservación de la memoria de las experiencias compartidas por los estudiantes sanmarquinos de pregrado, con lo que el LÉXICO SANMARQUINO se constituye en un antecedente para estudios lexicográficos en los que la unidad de análisis es la lengua juvenil; en específico, el léxico coloquial utilizado por estudiantes universitarios.

\section{REFERENCIAS BIBLIOGRÁFICAS}

1. Asociación de Academias de la Lengua Española (2010): Diccionario de americanismos, Santillana Ediciones Generales S. L: Lima.

2. Haensch, Günther y otros (1982). La lexicografía/De la lingüística teórica a la lexicografía práctica, Madrid: Gredos.

3. Lara, Luis (1990). Dimensiones de la lexicografía/A propósito del Diccionario del español de México, México D. F.: El Colegio de México.

4. Lara, Luis (2006). Curso de lexicología, México D. F.: El Colegio de México.

5. Martínez de Sousa, José (1995). Diccionario de lexicografía práctica, Barcelona: Biblograf.

6. Portilla, Luisa (2011). Léxico popular peruano/El habla de Lima, Lima: Universidad Ricardo Palma.

7. Porto-Dapena, José (2002). Manual de técnica lexicográfica, Madrid: Arco Libros.

8. Ramírez, Luis Hernán (1996). «Dialectos y lenguas especiales», en Estructura y funcionamiento del lenguaje, Lima: Derrama Magisterial.

9. Real Academia Española (2014). Diccionario de la lengua española, Barcelona: Espasa Libros, S. L. U.

CORRESPONDENCIA:

Luisa Portilla-Durand

lportillad@unmsm.edu.pe

FeCHAD DE RECEPCIÓN: 11-08-2020.

FECHA DE ACEPTACIÓN: 28-08-2020. 